\title{
ALOCAÇÃO ÓTIMA DE RECURSOS FINANCEIROS PARA MAXIMIZAR O NÍVEL DE SERVIÇO NO FORNECIMENTO DE UNIFORMES DA MARINHA DO BRASIL
}

\author{
Karina da Paz Bentes \\ Pontífice Universidade Católica do Rio de Janeiro (PUC-Rio) / Marinha do Brasil (MB) \\ Avenida Brasil, 10.500, Olaria, Rio de Janeiro - RJ \\ kabentes@hotmail.com \\ Bruno Fanzeres dos Santos \\ Pontífice Universidade Católica do Rio de Janeiro (PUC-Rio) \\ Rua Marquês de São Vicente, 225, Gávea, Rio de Janeiro - RJ \\ bruno.santos@puc-rio.br
}

\begin{abstract}
RESUMO
A atuação de forma eficiente e eficaz é fundamental na gestão orçamentária de órgãos públicos brasileiros, principalmente na atual situação econômica do País. Neste sentido, as Forças Armadas têm buscado ferramentas que as auxiliem na tomada de decisão e na aplicação de tais recursos. O objetivo do presente estudo é propor um modelo de alocação ótima de recursos financeiros, de forma a maximizar o nível de serviço, ou seja, elevar a disponibilidade do item no estoque, sob restrição orçamentária. A metodologia proposta foi aplicada a um caso de estudo real da Marinha do Brasil e os resultados demonstram que é possível aumentar significativamente a satisfação dos clientes com os recursos disponíveis, uma vez que a solução do modelo elevou em 50\% o nível de serviço, passando dos atuais $24 \%$ para $74 \%$. Além disso, a partir de uma modelagem complementar, constatou-se que um incremento médio de $8 \%$ no orçamento pode gerar melhorias contínuas no nível de serviço.
\end{abstract}

Palavras-chave: Recursos Públicos, Programação Linear, Nível de Serviço, Marinha do Brasil.

\begin{abstract}
An efficient and effective performance in budget-constrained management has become critical in public agencies in Brazil, especially due to the current economic situation of the country. In this sense, the Armed Forces have sought tools to assist them in decision making and in efficiently allocate of such resources. The objective of the present study is to propose an optimization model of optimal allocation of financial resources, in order to maximize the level of service, that is, to increase items availability in the inventory, in a budget-constrained. The methodology was applied in a real case of the Brazilian Navy and the results demonstrate that it is possible to significantly increase customer satisfaction with the available resources, since the solution of the model increased by $50 \%$ the level of service, going from the current $24 \%$ to $74 \%$. In addition, from a complementary modeling, it was found that an average $8 \%$ increase in the budget can generate continuous improvements in the level of service.
\end{abstract}


Keywords: Public Resources, Linear Programming, Service Level, Brazilian Navy.

\section{Como Citar:}

BENTES, Karina da Paz; SANTOS, Bruno Fanzeres dos. Alocação ótima de recursos financeiros para maximizar o nível de serviço no fornecimento de uniformes da Marinha do Brasil. In: SIMPÓSIO DE PESQUISA OPERACIONAL E LOGÍSTICA DA MARINHA, 19., 2019, Rio de Janeiro, RJ. Anais [...]. Rio de Janeiro: Centro de Análises de Sistemas Navais, 2019.

\section{INTRODUÇÃO}

O contexto econômico em que o Brasil está inserido, atualmente, revela, dentre outros aspectos, a dimensão dos problemas causados pela má administração dos recursos públicos por aqueles que deveriam zelar pela sua aplicação eficiente, uma vez que estes recursos, em sua maioria, são compostos por tributos pagos pela sociedade [12]. Segundo Trivelato et al. [24], em função de restrições orçamentárias, é de interesse de toda sociedade e dever dos gestores, respeitados os parâmetros técnicos e os recursos disponíveis, que o sistema opere com eficiência, transformando insumos em produtos e serviços.

Em virtude de os recursos em geral serem escassos, os princípios da especialidade e da focalização devem orientar o poder público na busca da satisfação dos interesses coletivos [2; 15]. A destinação dos recursos orçamentários precisa ser certeira, principalmente, para os fins assegurados na Constituição Federal [9]. De acordo com o Art. 37 da carta Magna, para que os gestores públicos alcancem o sucesso desejado na alocação dos recursos financeiros, é necessário observar, dentre outros, o princípio da eficiência [3]. Sendo assim, espera-se que a Administração possa aplicar seus recursos de maneira adequada de modo a produzir bons resultados, atendendo assim, às demandas da sociedade com maior celeridade [10].

No âmbito das Forças Armadas, compete ao Ministério da Defesa coordenar e consolidar o processo de alocação de recursos públicos, a partir das prioridades estabelecidas na Estratégia Nacional de Defesa (END). Conforme dados divulgados por este Órgão [6], nos últimos anos, houve um relevante incremento no capital destinado à defesa da Nação, passando de $\mathrm{R}$ \$ 59,5 bilhões em 2010, para R \$ 92,5 bilhões em 2017, correspondendo aproximadamente $1,4 \%$ do PIB brasileiro, sendo que destes 24,3 bilhões foram direcionados para a Marinha do Brasil (MB). Dentre as diversas finalidades de aplicação do orçamento recebido pela MB, encontra-se uma parcela reservada, exclusivamente, para aquisição de fardamento. De acordo com a Lei de Diretrizes Orçamentárias, estes recursos são caracterizados, por força de lei, como despesa obrigatória do Governo Federal, não podendo ser utilizados em outros propósitos. Este fato eleva, sobremaneira, a responsabilidade da MB em gerir com eficiência e eficácia tais recursos, uma vez que contribuem para a manutenção de seu estado de prontidão e de aprestamento para a defesa nacional.

É neste contexto que a Marinha do Brasil, vem buscando, constantemente, capacitar os seus gestores e aprimorar as técnicas voltadas para alocação ótima do orçamento, a fim de tornar a tomada de decisão mais eficiente e eficaz, de forma a contribuir para a melhoria contínua dos níveis de serviços oferecidos aos seus clientes. Portanto, este artigo tem como objetivo propor um modelo de alocação ótima de recursos financeiros, de forma a maximizar o nível de serviço, ou seja, elevar a disponibilidade do item em estoque, sob restrição orçamentária. Essa técnica possibilitará que a organização resolva o dilema entre a distribuição de recursos escassos e o atendimento da demanda de seus clientes, pois seus resultados, além de visar o alcance do melhor nível de serviço possível, indicarão a quantidade ótima a ser adquirida de cada item de fardamento, eliminando possíveis perdas e desperdícios. 
Para tanto, este artigo está composto por uma breve introdução, seguida de uma revisão bibliográfica sobre o tema. Na terceira seção é apresentada a metodologia utilizada, enquanto a quarta seção demonstra os modelos propostos, seguida dos resultados obtidos. E por fim, é apresentada uma conclusão geral sobre o tema em lide.

\section{REFERENCIAL TEÓRICO} estudo.

Esta seção apresenta a revisão bibliográfica que serve de base para a elaboração desse

\subsection{EFICIÊNCIA E EFICÁCIA NA ALOCAÇÃO DOS RECURSOS ORÇAMENTÁRIOS}

Historicamente, a preocupação com maior eficiência na Administração Pública iniciou-se na década de 80 com os movimentos pela Reforma do Estado ocorrida nos países anglo-saxões, particularmente nos EUA de Ronald Reagan e na Inglaterra com Margareth Thatcher, propagando para outros países, inclusive o Brasil, na década de 90 [16]. Essa preocupação ganhou força, no início dos anos 2000, com a criação da Lei de Responsabilidade Fiscal (LRF). Segundo Soares e Scarpin [23], a LRF veio coroar, por meio de normas, toda a ação da administração pública, pois passou a exigir o estabelecimento de metas, prioridades e eficiência, fazendo com que a atuação do gestor esteja voltada para o atendimento das necessidades da sociedade, sem perder de vista o equilíbrio fiscal.

Santos [22] declara que o orçamento público demonstra a capacidade e a qualidade do planejamento e da gestão pública na sua totalidade - seja em gestão de pessoas, de processos, de projetos ou de informações -, bem como a respectiva administração do patrimônio. Para a autora, um "orçamento bem elaborado e executado é sinalizador de uma gestão pública de qualidade”.

Segundo De Carvalho Fer e Lima [9], o orçamento pode ser entendido como um pacto de confiança firmado entre o povo e o seu governante, para que este, em nome da sociedade, possa realizar o dispêndio dos recursos públicos. Sendo assim, sua alocação deve ser a mais eficiente e eficaz possível. A rapidez com que as informações são geradas e compartilhadas, atualmente, faz com que a sociedade passe a ser mais atuante na fiscalização das ações dos gestores públicos.

Uma ferramenta que veio corroborar para o aumento da participação do cidadão no controle da aplicação dos recursos orçamentários, no âmbito do Governo Federal, é o Portal da Transparência [4]. Através deste canal, todos podem acompanhar a utilização dos recursos federais arrecadados com impostos no fornecimento de serviços públicos à população.

Para Soares e Scarpin [23], "o princípio da eficiência norteia a atividade administrativa no sentido de obter os melhores resultados com os meios escassos de que se dispõe, e a menor custo.” Sendo assim, torna-se importante diferenciar eficiência de eficácia.

Enquanto a eficiência está voltada para os métodos e processos depreendidos para se alcançar determinados resultados, a eficácia refere-se aos objetivos e resultados atingidos [7]. Luchi e Carneiro [20], acrescentam concluindo como procedimento eficiente o uso adequado de recurso disponível para concretização de um objetivo e como procedimento eficaz, aquele que atinge os efeitos esperados e metas a que se propôs.

Ademais, é importante salientar que o conceito de eficiência na administração pública vai além do significado normativo da palavra, pois assume-se que os gestores públicos se preocupam não só com o cumprimento das leis e com a correta aplicação dos recursos públicos, mas também com a otimização do gasto público, ou seja, com o verdadeiro atendimento às demandas da sociedade [23].

\subsection{NÍVEL DE SERVIÇO.}


De acordo com Coutinho [8], os fundamentos da nova administração pública voltada para o cidadão mostram que, se a qualidade dos serviços depende da satisfação do usuário, então, o seu atendimento deve ser sempre o melhor possível.

Hayes [18] enfatiza que a satisfação do cliente é algo primordial e pode ser definida como a resposta da impressão do cliente acerca da capacidade de uma organização em identificar e atender às suas necessidades de forma eficiente. Neste sentido, quando uma organização consegue gerar valor aos seus clientes e ofertar produtos e serviços que superem ou equilibrem as suas expectativas, trata-se de nível de serviço logístico [11].

Kovacs [19] acrescenta que dentre os principais atributos que compõem o serviço ao cliente, encontra-se a disponibilidade, ou seja, a capacidade de ter estoques quando o produto é desejado pelo cliente. Gallmann e Belvedere [14], ao pesquisarem sobre como as empresas com melhor desempenho se esforçam para melhorar níveis de serviço, perceberam que um grande desafio para as organizações é garantir a disponibilidade de produtos aos clientes sem que ocorra custos extras. Para isso, elas necessitam ter pleno conhecimento das variáveis que possam influenciar positivamente o nível de serviço.

Segundo Fleury et al. [13], as organizações devem procurar um equilíbrio na manutenção dos estoques de forma a obter um estoque mínimo que compense os custos derivados das atividades de produção e abastecimento, ao mesmo tempo que atenda ao nível de serviço desejado pelos clientes.

\section{PROCEDIMENTOS METODOLÓGICOS PARA A CONSTRUÇÃO DO MODELO}

A pesquisa foi realizada na Gerência de Fardamento do Centro de Controle de Inventário da Marinha (CCIM), organização militar subordinada a Diretoria de Abastecimento da Marinha, responsável por gerir os recursos orçamentários destinados a aquisição de itens das diversas categorias de material sob sua supervisão, dentre elas o fardamento. Compete a Gerência de Fardamento, dentre outras funções, executar as atividades gerenciais "Determinação Corrente de Necessidades" e "Controle de Inventário"; promover as atividades gerenciais "Obtenção" e "Destinação de Excessos"; e manter adequado estoque de peças de uniformes no Depósito de Fardamento da Marinha no Rio de Janeiro (DepFMRJ), assegurando o fluxo contínuo de fornecimento aos Postos de Distribuição de Uniformes (PDU) e de Encomenda de Uniformes (PEU) [5].

Visando atingir os objetivos do estudo, esta pesquisa cumpriu os passos metodológicos definidos por Arenales, et. al. [1] e considerou, como premissa, a sequência de etapas descritas na Figura 1. Cabe ressaltar, que podem haver ciclos entre essas fases, uma vez que um modelo matemático nem sempre é formulado de uma só vez, pois equívocos e inconsistências podem ocorrer durante a sua execução, sinalizando a necessidade de revisão do modelo [1].

O problema foi construído a partir de entrevistas não estruturadas com o Gerente de Fardamento do CCIM e seus auxiliares, que o apoiam no planejamento da obtenção de itens de fardamento fornecidos pelo Sistema de Abastecimento da Marinha (SAbM). Em seguida, foram realizados levantamentos bibliográficos que serviram de base para a elaboração do referencial teórico. 
Formulação do problema;

Identificação e coleta de dados;

Construção do modelo;

Solução do modelo utilizando linguagem de programação;

Validação do modelo com a realização de experimento piloto;

Realização de ajustes no modelo e coleta de novos dados;

Implementação da solução com a execução da otimização; e

\section{Análise dos resultados.}

Figura 1 - Etapas da pesquisa

Fonte: Adaptado de Arenales et. al., (2015).

O próximo passo na formulação do problema foi a análise da sistemática atual empregada na ferramenta "Plano de Obtenção", utilizada para executar o planejamento das compras de fardamento a partir da distribuição dos recursos orçamentários disponíveis pelos diversos itens existentes nesta categoria de material. Constatou-se, então, que um dos maiores desafios da Gerência de Fardamento do CCIM é adequar o recurso recebido, anualmente, com as demandas dos itens sob sua responsabilidade. O planejamento da obtenção executado por esta gerência, visa o atendimento das demandas de dois anos consecutivos, ou seja, no ano "A", planeja-se formar estoque para atender à demanda do ano “ $A+1$ ” e à manutenção do estoque de segurança.

Os dados coletados no Plano de Obtenção de 2018 mostraram que os recursos recebidos são insuficientes para atender $100 \%$ da demanda de todos os itens. Sendo assim, visando garantir, ao menos, a demanda dos itens mais críticos, segundo a modalidade de fornecimento, que são os destinados aos militares que estão incorporando na MB, o Gerente de Fardamento precisou adotar algumas medidas, como por exemplo, fixar como política de compra a aquisição de apenas 30\% da quantidade demandada para todos os itens, com exceção dos insumos no exterior, o que tem gerado inúmeros problemas, tais como: quebra de estoque; priorização de atendimento de alguns militares em detrimento de outros; baixo nível de serviço nos PDU; baixa adesão dos militares em adquirir os itens no SAbM devido à ausência da maioria dos itens em estoque; e falta de credibilidade no SAbM.

Como o Plano de Obtenção da Gerência de Fardamento está pautado em uma planilha do Excel, ele apresenta algumas deficiências e limitações. Em que pese a utilização de fórmulas matemáticas na construção das quantidades a serem compradas de cada item, na maioria das vezes é necessário que os operadores desta planilha atuem de forma manual no ajuste destas quantidades, para que o valor total esteja compatível com o orçamento disponível. Assim, vislumbrou-se a grande necessidade de se construir um modelo de programação matemática que aperfeiçoasse este planejamento.

Após a compreensão e a formulação do problema, identificou-se os elementos essenciais para a construção da modelagem, como: demanda, preço, quantidade em estoque, quantidades em dívida e estoque de segurança. Neste momento, percebeu-se que a demanda era uma variável complexa, o que exigiria uma maior atenção, pois ela é composta por três categorias distintas, diferenciadas pela modalidade de fornecimento:

- Incorporação: itens fornecidos de forma gratuita aos militares que estão ingressando na Marinha do Brasil; 
- Credifarda: itens fornecidos aos militares da ativa de graduação inferior a terceirosargento, ou seja, a cabos, marinheiros e soldados e, em casos especiais, a outros militares, conforme créditos disponibilizados, anualmente, a estes militares; e

- Venda particular: itens fornecidos a quaisquer militares da ativa, mediante indenização por meio de desconto em bilhete de pagamento do militar.

A quantidade de produtos que compõem o catálogo de fardamento da $\mathrm{MB}$, fornecidos pelo SAbM, gira em torno de 3.300 (três mil e trezentos) produtos, sendo 98\% deles adquiridos no Brasil. As Organizações Militares (OM) que integram o SAbM, utilizam na condução de suas atividades, um sistema computacional integrado denominado SINGRA - Sistema de Informações Gerenciais do Abastecimento.

A coleta de dados sobre quantidade de itens, descrição, andainas de incorporação e de credifarda, nível de estoque, preço, demandas (incorporação, credifarda e venda particular) e quantidades em dívida, foi realizada no banco de dados do SINGRA. Utilizou-se como referência o ano de 2018. Visando manter uma coerência entre os dados trabalhados com o problema real, buscou-se o alinhamento dos mesmos com as informações contidas no Plano de Obtenção de 2018. O estoque de segurança foi coletado deste plano de obtenção.

Em seguida foi construído um modelo matemático de Pesquisa Operacional baseado em Programação Linear Inteira Mista, com o objetivo de maximizar o nível de serviço a partir da alocação ótima de recursos, sob restrição orçamentária, cuja função objetivo visa maximizar o nível de serviço oferecido aos militares que utilizam o SAbM como fonte de aquisição de seus uniformes.

O modelo foi desenvolvido no IDE Juno for JuliaPro e executado na plataforma JuliaPro 0.6.3.1. A otimização foi realizada por meio do Solver Gurobi, em um processador Intel i7-2600 CPU 3.40GHz e 3.40GHz com 16GB RAM. Arquivos em Microsoft Excel com a extensão “.csv" foram criados para armazenamento dos dados de entrada do modelo.

Após a compilação do modelo e o ajuste das variáveis envolvidas, foi realizado um teste piloto com apenas vinte produtos, escolhidos de forma aleatória, a fim de averiguar se o resultado obtido atenderia aos pressupostos do sistema real. Com isso, verificou-se que ambos sistemas (matemático e o real) convergem de forma harmoniosa e que a solução proposta atinge o objetivo esperado.

Os resultados do teste piloto foram apresentados ao Gerente de Fardamento do CCIM. Após a sua avaliação, observou-se que os dados de entrada do nível de estoque e das demandas necessitavam de ajustes. O nível de estoque passou a compreender o somatório das quantidades disponíveis em estoque, comprometidas, em arrecadação, inscritas nos pedidos de obtenção e em ordens de compra. As demandas foram compostas pela média aritmética dos últimos 3 anos, ou seja, 2015 a 2017. Com isso, novas extrações foram realizadas no SINGRA. Contudo, não houve a necessidade de alterar a modelagem inicial do problema.

Visando ampliar o estudo, uma modelagem complementar foi proposta, onde se pretende, a partir de um nível de serviço fixo, previamente estabelecido, revelar a quantidade mínima de recursos financeiros necessários para atingir este nível de serviço. Sendo assim, a função objetivo passou a focar na minimização do orçamento. Novos testes foram realizados, tendo como base os 20 itens do teste piloto a fim de validar os modelos. As soluções demonstraram que ambos os modelos ( 1 - inicial e 2 - complementar) representam apropriadamente o problema em estudo e mantém a integridade das características do sistema real.

A partir da validação dos modelos, o próximo passo foi a implementação da solução em um estudo completo, com a execução da otimização dos 3.300 itens de fardamento que compõem a carteira de produtos fornecidos pelo SAbM. Utilizou-se como base orçamentária os recursos disponíveis em 2018 para aquisição destes itens, ou seja, R\$ 21.248.781,00 (vinte e um milhões, duzentos e quarenta e oito mil, setecentos e oitenta e um reais). Por fim, os re- 
sultados foram analisados e comparados com a solução gerada pelo Plano de Obtenção de 2018.

\section{FORMULAÇÃO DOS MODELOS}

\subsection{MODELO 1 - INICIAL}

\subsubsection{Função Objetivo}

Segundo Miguel et. al. [21], o desenvolvimento de modelos pode auxiliar na compreensão do ambiente em estudo e seus problemas, ajudando a tomada de decisão. A modelagem matemática, além de representar de forma apropriada o problema da vida real, deve possibilitar sua resolução por meio de métodos de solução disponíveis e com esforços computacionais aceitáveis [17]. Dessa forma, o modelo matemático, baseado em Programação Linear Inteira Mista, desenvolvido para o auxílio na tomada de decisão para a alocação ótima de recursos, sob restrição orçamentária, visando maximizar o nível de serviço, foi:

$$
\text { Função Objetivo: } \quad \text { Max } \delta
$$

Sendo, $\delta$ definida como nível de serviço.

\subsubsection{Restrições Impostas ao Modelo}

\subsubsection{1.}

Orçamento Disponível

A primeira restrição condiciona que o somatório das quantidades totais a serem adquiridas, por item, multiplicadas pelos seus respectivos valores unitários, seja igual ou inferior ao orçamento disponível, conforme expresso na inequação abaixo:

$$
\sum_{i=1}^{n}\left(P_{i} i Q_{i}\right) \leq B i
$$

Sendo:

$$
\begin{aligned}
& \mathrm{n}=\text { Número de itens considerados; } \\
& \mathrm{P}_{\mathrm{i}}=\text { Preço unitário por item; } \\
& \mathrm{Q}_{\mathrm{i}}=\text { Quantidade total por item; e } \\
& \mathrm{B}=\text { Orçamento. }
\end{aligned}
$$

\subsubsection{2.}

\section{Nível de Serviço}

A segunda restrição estabelece a inequação que determinará o nível de serviço. Para isso, adotou-se como nível de serviço o resultado da quantidade total adquirida adicionada à quantidade disponível em estoque, divididas pelo somatório das três demandas, multiplicadas por dois, a fim de atender a dois anos consecutivos, com as quantidades em dívida e necessárias para formar o estoque de segurança. A inequação (3) demonstra a referida restrição.

$$
\delta \leq \frac{\left(Q_{i}+E_{i}\right)}{\left(2\left(D_{i}+D_{i}+D_{i}+\text { Part }_{i}\right)+\dot{\epsilon}_{i}+E S_{i}\right)}, \forall i=1, \ldots, n
$$


Sendo:

$\mathrm{n}$ = Número de itens considerados;

$\mathrm{Q}_{\mathrm{i}}=$ Quantidade total por item;

$\mathrm{E}_{\mathrm{i}}=$ Quantidade disponível em estoque por item;

$\mathrm{DIC}_{\mathrm{i}}=$ Demanda de incorporação por item;

$\mathrm{DCF}_{\mathrm{i}}=$ Demanda de credifarda por item;

DPart $_{\mathrm{i}}=$ Demanda de venda particular por item;

$\operatorname{Div}_{\mathrm{i}}=$ Quantidade em dívida por item; $\mathrm{e}$

$\mathrm{ES}_{\mathrm{i}}=$ Estoque de segurança por item.

Seguindo a inequação (3) e a função objetivo (1), ressalta-se que está sendo buscado a maximização do menor nível de serviço dentre todos os itens considerados. Desta forma, assegura-se que o nível de serviço de todos os itens será superior ao valor ótimo identificado pelo modelo.

\subsubsection{Demanda da Incorporação}

Dentre as três principais modalidades de fornecimento de uniformes (incorporação, credifarda e venda particular), há uma premissa na Gerência de Fardamento de que a demanda da incorporação tem prioridade de atendimento em detrimento das outras, ou seja, o nível de serviço para as incorporações deve ser de $100 \%$. Com isso, criou-se a seguinte restrição:

$$
\left(Q_{i}+E_{i}\right) \geq D I C_{i}, \forall i=1, \ldots, n
$$

Sendo assim, a quantidade total a ser comprada, por item, acrescida da quantidade existente no estoque deve, pelo menos, garantir o atendimento das necessidades das incorporações. Outra política adotada na Gerência de Fardamento é a aquisição plena de todos os insumos obtidos no Exterior, pois são matérias primas essenciais para a fabricação de diversos outros itens de fardamento. Portanto, a fim de garantir que estes insumos também tenham um nível de serviço máximo de $100 \%$, as demandas destes itens foram enquadradas como demandas de incorporação.

\subsubsection{Nível de Serviço Máximo}

Conforme demonstrado, abaixo, a última restrição fixa um limite máximo para a variável nível de serviço, ou seja, $100 \%$.

$$
\delta \leq 1
$$

\subsubsection{Modelo Compilado}

Desse modo, o problema de otimização foi estruturado da seguinte forma:

Função objetivo: $\max _{\delta, Q_{i}}$

Sujeito a:

$$
\begin{gathered}
\sum_{i=1}^{n}\left(P_{i} \dot{i} Q_{i}\right) \leq B i \\
\delta \leq \frac{\left(Q_{i}+E_{i}\right)}{\left(2\left(D I C_{i}+D C F_{i}+\text { DPart }_{i}\right)+\dot{i}_{i}+E S_{i}\right)}, \forall i=1, \ldots, n
\end{gathered}
$$




$$
\begin{gathered}
\left(Q_{i}+E_{i}\right) \geq D I C_{i}, \forall i=1, \ldots, n \\
0 \leq \delta \leq 1 \\
Q_{i} \in Z_{+i, \forall i=1, \ldots, n i}
\end{gathered}
$$

\subsection{MODELO 2 - COMPLEMENTAR}

\subsubsection{Função Objetivo}

Conforme descrito na seção 3 deste artigo, com o objetivo de ampliar o estudo, e assim, contribuir para a melhoria da tomada de decisão por parte do gestor da cadeia de fardamento do CCIM, uma modelagem complementar foi proposta. Nela pretende-se, a partir de um nível de serviço fixo, previamente estabelecido, revelar a quantidade mínima de recursos financeiros necessários para atingir este nível de serviço. Sendo assim, a modelagem complementar proposta para minimizar o orçamento foi:

Função Objetivo: $\quad$ Min B2

Sendo, B2 = orçamento necessário para atingir o nível de serviço desejado.

\subsubsection{Restrições Impostas ao Modelo}

As restrições do modelo complementar foram idênticas às do modelo inicial, com exceção da inequação (5) que foi retirada, ocorrendo apenas a alteração das variáveis Q para Q2, B para B2 e $\delta$ para NS, a fim de diferenciar um modelo do outro e possibilitar a execução computacional dos mesmos. Se anteriormente, o orçamento (B) era um parâmetro e o nível de serviço $(\delta)$ uma variável de decisão, na modelagem complementar estes papéis se inverteram. O nível de serviço (agora escrito como NS) passou a ser parâmetro e o orçamento, uma variável de decisão.

\subsubsection{Modelo Compilado}

Portanto, o modelo de otimização complementar a este estudo, foi escrito da seguinte forma:

Função objetivo: $\min B 2$

Sujeito a:

$$
\begin{gathered}
\sum_{i=1}^{n}\left(P_{i} \dot{i} Q 2_{i}\right) \leq B 2 i \\
N S \leq \frac{\left(Q 2_{i}+E_{i}\right)}{\dot{i} \dot{l}} \\
\left(Q 2_{i}+E_{i}\right) \geq D I C_{i}, \forall i=1, \ldots, n \\
B 2 \geq 0 \\
Q_{i} \in Z_{+i, \forall i=1, \ldots, n i}
\end{gathered}
$$


Sendo:

$\mathrm{n}$ = Número de itens considerados;

$\mathrm{P}_{\mathrm{i}}=$ Preço unitário por item;

$\mathrm{Q}_{\mathrm{i}}=$ Quantidade total por item;

NS = Nível de serviço pretendido;

$\mathrm{E}_{\mathrm{i}}=$ Quantidade disponível em estoque por item;

$\mathrm{DIC}_{\mathrm{i}}=$ Demanda de incorporação por item;

$\mathrm{DCF}_{\mathrm{i}}=$ Demanda de credifarda por item;

DPart $_{i}=$ Demanda de venda particular por item;

$\operatorname{Div}_{\mathrm{i}}=$ Quantidade em dívida por item; e

$\mathrm{ES}_{\mathrm{i}}=$ Estoque de segurança por item.

Ressalta-se que o nível de serviço (NS) neste modelo é um nível mínimo imposto a todos os itens considerados, portanto garantindo total eficiência do sistema.

\section{RESULTADOS E DISCUSSÕES.}

\subsection{MODELO 1 - INICIAL}

Respeitando-se todas as restrições, a solução gerada pelo programa foi considerada ótima, significando que a função objetivo pôde ser maximizada. Além de otimizar a alocação do orçamento, revelando a quantidade ideal a ser adquirida de cada item de fardamento, eliminando possíveis perdas e desperdícios, atingiu-se um nível de serviço mínimo de 74\%.

A Tabela 1 traz a comparação da quantidade de itens de fardamento classificados segundo os níveis de serviço gerados pelo Plano de Obtenção de 2018 e pelo modelo proposto. Primeiramente, ressalta-se que os 24 itens distribuídos no intervalo de 0 a $10 \%$ referem-se a uniformes que ainda não foram fornecidos pelo SAbM, não possuindo estoque nem registro de demanda. Assim, verificou-se que o menor nível de serviço do atual Plano de Obtenção é $24 \%$ e que 278 itens estão entre este percentual e $70 \%$. Ademais, dentre os que estão classificados na faixa de $70 \%$ a $80 \%$, 21 itens ainda se encontram abaixo do percentual mínimo conseguido com o modelo proposto, ou seja, são menores que $74 \%$.

Portanto, quando se compara os níveis de serviço obtidos no Plano de Obtenção da Gerencia de Fardamento do CCIM, com o extraído do modelo, constata-se que o modelo proposto, além de considerar o atendimento das demandas de incorporação para todos os itens e dos insumos adquiridos no Exterior em sua plenitude, ou seja, 100\% de satisfação, fez com que o menor nível de serviço tivesse um acréscimo de 50\%, saindo dos atuais $24 \%$ e chegando ao patamar de $74 \%$, utilizando o mesmo orçamento disponível. Com isso, garantiu-se que nenhum item terá um atendimento menor do que a solução ótima gerada pelo modelo. 


\begin{tabular}{|c|c|c|}
\hline \multirow{2}{*}{$\begin{array}{c}\text { NÍVEL DE } \\
\text { SERVIÇO }\end{array}$} & $\begin{array}{c}|c| \\
\text { QUANTIDADE DE ITENS } \\
\text { TENÇÃO }\end{array}$ & $\begin{array}{c}\text { MODELO PRO- } \\
\text { POSTO }\end{array}$ \\
\hline $0-10 \%$ & 24 & 0 \\
\hline $10 \%-20 \%$ & 0 & 0 \\
\hline $20 \%-30 \%$ & 41 & 0 \\
\hline $30 \%-40 \%$ & 16 & 0 \\
\hline $40 \%-50 \%$ & 44 & 0 \\
\hline $50 \%-60 \%$ & 86 & 0 \\
\hline $60 \%-70 \%$ & 91 & 0 \\
\hline $70 \%-80 \%$ & 95 & 852 \\
\hline $80 \%-90 \%$ & 139 & 155 \\
\hline $90 \%-100 \%$ & 2763 & 2292 \\
\hline
\end{tabular}

Fonte: Os autores.

A Tabela 2 demonstra as principais diferenças entre a ferramenta de planejamento de obtenção usada na Gerência de Fardamento e o modelo de programação linear inteira mista proposto neste artigo:

Tabela 2 - Principais diferenças entre as ferramentas de planejamento da obtenção

\section{PLANO DE OBTENÇÃO 2018}

Ferramenta simples e primária.

Não considera demandas separadas.

Há intervenção manual do operador no ajuste das quantidades.

*Menor nível de serviço: $24 \%$.

Problemas na distribuição dos recursos. A solução proposta não é ótima, pois aplica-se dinheiro onde não precisa e deixa-se de aplicar onde necessita.

Demora na obtenção dos resultados.

* Ambos calculados com o mesmo orçamento.

Fonte: Os autores.

Visando exemplificar a atuação do modelo, explicando alguns dos resultados sugeridos na otimização, foi confeccionada a Tabela 3, com 10 itens mais representativos em relação as diferenças entre os resultados, sendo cinco itens com a solução do modelo indicando a redução da quantidade a ser adquirida em relação à proposta no Plano de Obtenção de 2018 e outros cinco itens com a solução do modelo incentivando o aumento da quantidade. Assim, verifica-se que os itens cujas quantidades foram reduzidas, se devem ao fato de que, na maioria das vezes, os estoques são suficientes para atender dois anos da demanda da incorporação, mais um ano das demandas de credifarda e venda particular, além da quantidade em dívida e do estoque de segurança, não sendo necessário comprar o item, como é o caso da CALÇA AZUL-MESCLA 42.

Já para os itens cujas quantidades foram elevadas, após a análise dos dados que deram origem a esta solução, observou-se que este aumento justifica-se, na grande maioria dos casos, porque a quantidade sugerida pelo Plano de Obtenção de 2018 somada a quantidade disponível em estoque, é insuficiente para atender a demanda total de um ano, nem mesmo con- 
seguiria cobrir o estoque de segurança e a dívida, como pode ser visto no item GORRO CAMUFLADO. Assim, para este item específico, com a solução gerada pelo modelo, poder-se-á atender dois anos da demanda de incorporação, um ano e meio do credifarda, mais um ano da venda particular, além do estoque de segurança e da quantidade em dívida.

Tabela 3 - Comparação entre a solução proposta pelo Plano de Obtenção 2018 e pelo Modelo de otimização.

\begin{tabular}{|c|c|c|c|c|c|c|c|c|c|}
\hline NOME_ITEM & $\begin{array}{c}\text { QTD } \\
\text { DÍVIDA }\end{array}$ & $\begin{array}{l}\text { EST. } \\
\text { SEG }\end{array}$ & $\begin{array}{c}\text { ESTO } \\
\text { QUE }\end{array}$ & $\begin{array}{c}\text { DEM } \\
\text { INCORP }\end{array}$ & $\begin{array}{c}\text { DEM } \\
\text { CREDIF }\end{array}$ & $\begin{array}{l}\text { DEM } \\
\text { PART }\end{array}$ & $\begin{array}{c}\text { DEMAN } \\
\text { DA } \\
\text { TOTAL }\end{array}$ & $\begin{array}{c}\text { RESUL } \\
\text { TADO } \\
\text { PLANO } \\
\text { OBT. }\end{array}$ & $\begin{array}{l}\text { RESUL } \\
\text { TADO } \\
\text { MODE } \\
\text { LO }\end{array}$ \\
\hline $\begin{array}{l}\text { AGASALHO } \\
\text { AZUL DE } \\
\text { FRIO M }\end{array}$ & 269 & 88 & 2255 & 301 & 54 & 895 & 1250 & 181 & 0 \\
\hline $\begin{array}{l}\text { CALCA } \\
\text { AZUL- } \\
\text { MESCLA } 42\end{array}$ & 3102 & 463 & 13635 & 2790 & 2851 & 959 & 6600 & 939 & 0 \\
\hline $\begin{array}{l}\text { CALCA } \\
\text { CAMUFLAD } \\
\text { A } 40\end{array}$ & 1441 & 442 & 5833 & 1738 & 2900 & 1662 & 6300 & 2595 & 4828 \\
\hline $\begin{array}{l}\text { CAXANGA } \\
57\end{array}$ & 3756 & 568 & 16099 & 3126 & 4380 & 594 & 8100 & 1328 & 0 \\
\hline $\begin{array}{l}\text { GANDOLA } \\
\text { CAMUFLAD } \\
\text { A } 2\end{array}$ & 1446 & 793 & 9255 & 2058 & 5638 & 3604 & 11300 & 4675 & 9029 \\
\hline $\begin{array}{l}\text { GORRO } \\
\text { CAMUFLAD } \\
\mathrm{O}\end{array}$ & 2670 & 1157 & 7275 & 3364 & 7146 & 5990 & 16500 & 8866 & 19832 \\
\hline $\begin{array}{l}\text { CALCAO } \\
\text { AZUL } \\
\text { GINASTICA } \\
\text { PRAÇA M }\end{array}$ & 9091 & 1157 & 31491 & 6120 & 5877 & 4503 & 16500 & 3527 & 343 \\
\hline $\begin{array}{l}\text { SAPATO } \\
\text { BRANCO } 42\end{array}$ & 366 & 119 & 1497 & 123 & 171 & 1406 & 1700 & 716 & 1363 \\
\hline MEIA PRETA & 33633 & 7014 & 112056 & 29972 & 47192 & 22836 & 100000 & 38577 & 65076 \\
\hline $\begin{array}{l}\text { TOALHA } \\
\text { BANHO }\end{array}$ & 13532 & 3114 & 78416 & 14851 & 13522 & 16027 & 44400 & 8109 & 0 \\
\hline
\end{tabular}

Fonte: Os autores.

\subsection{MODELO 2 - COMPLEMENTAR}

A solução gerada pelo programa para o modelo 2 foi considerada ótima, pois a função objetivo foi minimizada. Primeiramente, executou-se a otimização fixando um nível de serviço inicial de 70\%, alcançando um orçamento mínimo de R\$ 18.894.745. Posteriormente, o modelo foi executado por diversas vezes, sempre acrescendo 3\% ao nível de serviço mínimo desejado para todos os itens, até chegar ao patamar de 100\%. Os valores orçamentários encontrados estão demonstrados na Figura 2, a seguir: 


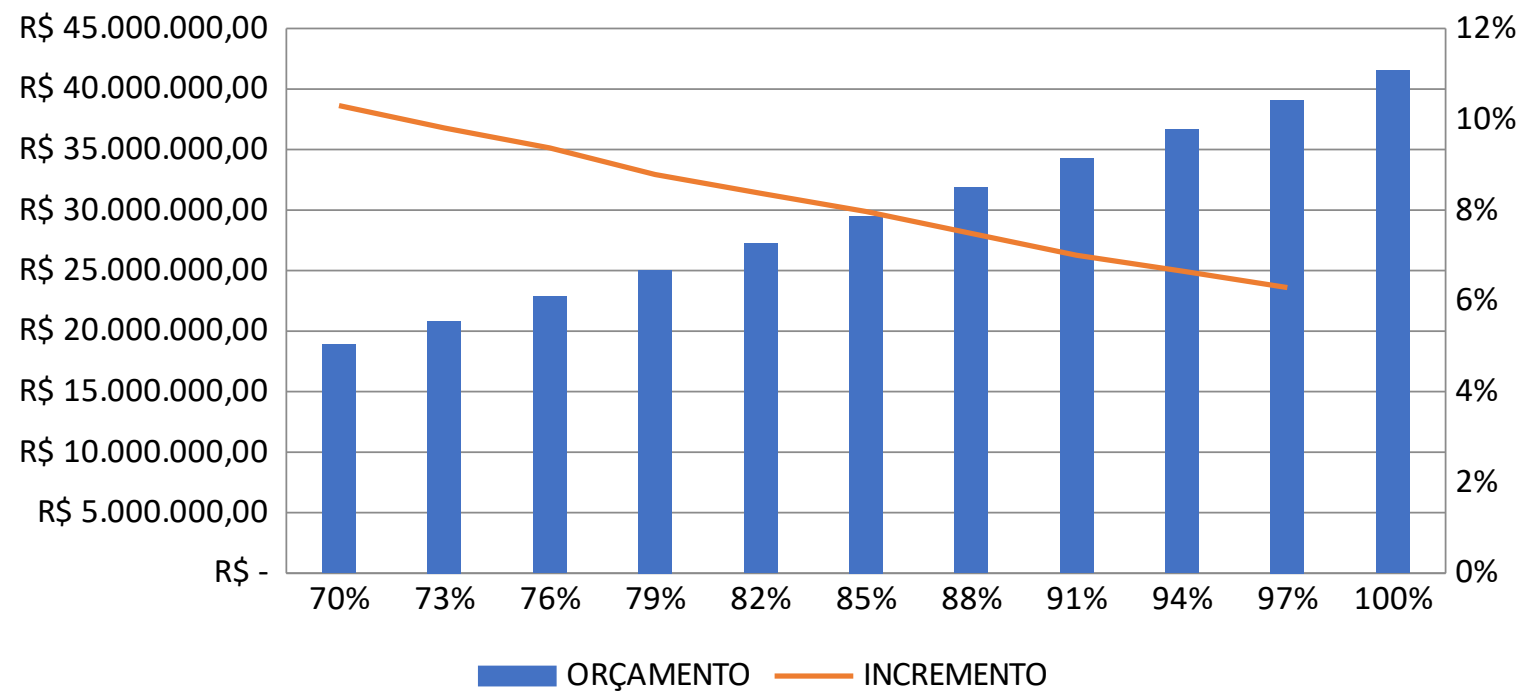

Figura 2 - Gráfico do Orçamento mínimo x Nível de serviço desejado Fonte: Os autores.

Os resultados obtidos demonstram que o modelo complementar, proposto, atingiu o objetivo pretendido e se configurou uma excelente ferramenta para auxiliar a tomada de decisão, uma vez que revela o orçamento mínimo necessário para conquistar um determinado nível de serviço. Sendo assim, cabe ressaltar que, no problema em estudo, as soluções apontam que com um pequeno incremento no orçamento consegue-se aumentar o percentual do nível de serviço, ou seja, com apenas $10 \%$ a mais no orçamento, passa-se de $73 \%$ para $76 \%$ de nível de serviço. Essa elevação, embora seja pequena, gera diversos efeitos positivos na cadeia logística, uma vez que o aumento da satisfação dos clientes revela a eficiência com que a organização administra os seus recursos.

\section{CONCLUSÃO}

O objetivo principal deste estudo foi a aplicação prática de um modelo de alocação ótima de recursos financeiros, de forma a maximizar o nível de serviço, ou seja, elevar a disponibilidade do item no estoque, sob restrição orçamentária. Pode-se dizer que o mesmo foi atingido, uma vez que o modelo desenvolvido pôde ser otimizado através de um software e executado em um caso real de aplicação de recursos nas compras de itens de fardamento da Marinha do Brasil.

Devido à grande importância da utilização dos recursos públicos, a ideia central deste trabalho consistiu em analisar como está sendo realizada a aplicação do orçamento na Gerência de Fardamento do CCIM, efetuada através do Plano de Obtenção de 2018, e respeitando as suas principais características, verificar como a Pesquisa Operacional poderia propor melhorias a este planejamento. O desafio foi saber se a modelagem baseada em Programação Linear Inteira Mista é uma ferramenta eficiente para a resolução deste problema. Ao implementar a solução do modelo foi possível constatar algumas vantagens, como por exemplo, a considerável elevação do nível de serviço, uma vez que ficou maior do que o dobro do valor inicial. Outra vantagem observada foi a rapidez na obtenção dos resultados, bem como, a distribuição dos recursos ter sido feita de forma automatiza e otimizada.

Notou-se, também, que as diferenças entre as soluções propostas pelo Plano de Obtenção de 2018 e pelo modelo, sejam elas aumentando ou diminuindo as quantidades de compra, baseiam-se nas limitações existentes na ferramenta que sustenta o Plano de Obtenção, uma vez que não utiliza técnicas de otimização em sua operação. O cálculo das quantidades, embo- 
ra seja feito por meio de fórmulas matemáticas, é realizado de forma isolada por item, não considerando a composição de todos os itens de fardamento que compõem a planilha.

Como limitação encontrada temos a rigidez dos resultados, pois por ser um modelo determinístico, não possibilita a inclusão de variáveis que assumam valores incertos, podendo fazer com que as soluções não sejam mais viáveis, caso imprevistos aconteçam. Em relação às expectativas futuras, sugere-se novos estudos que: aumentem a complexidade do modelo, incluindo variáveis incertas traduzidas em modelos estocásticos; calculem o custo de venda perdida, decorrente da ruptura de estoque; e que possam definir, baseado nos históricos de demanda, quais os itens que devam ser mantidos em estoque pela MB e quais poderiam ficar armazenados com os fornecedores.

\section{REFERÊNCIAS BIBLIOGRÁFICAS}

[1] ARENALES, M. et al. Pesquisa operacional: para cursos de engenharia. Elsevier Brasil, 2015.

[2] BIDERMAN, C. et al. Um estudo considerando a "guerra fiscal" como elemento de interação na renda tributária dos estados brasileiros entre 1988/1998. Série para Discussão Cepesp, GV Pesquisa (NPP), n. 03, 2004.

[3] BRASIL, Constituição da República Federativa do Brasil de 1988. Disponível em: http://www.planalto.gov.br/ccivil 03/constituicao/constituicaocompilado.htm Acesso em: 26dez2018.

[4] BRASIL, Controladoria-Geral da União. Portal da Transparência. Disponível em: http://www.portaltransparencia.gov.br Acesso em 02abr2019.

[5] BRASIL, Ministério da Defesa. Marinha do Brasil. Secretaria Geral da Marinha. SGM- 201: Normas para Execução do Abastecimento. 6ª rev. Brasília, DF. 2009.

[6] BRASIL, Ministério do Planejamento, Desenvolvimento e Gestão. Secretaria de Orçamento Federal. Manual técnico de orçamento. Edição 2018. Brasília, 2018. www.planejamento.gov.br/assuntos/orcamento-1/informacoes.../MTOs/mto atual.pdf Acesso em 27dez2018.

[7] CATELLI, A.; SANTOS, E. S. Mensurando a Criação de Valor na Gestão Pública. Revista de Administração Pública. Rio de Janeiro, v. 38, n.3, p. 423- 450, 2004.

[8] COUTINHO, M. J. V. Administração pública voltada para o cidadão: quadro teórico-conceitual. Revista do Serviço Público, v. 51, n. 3, p. 40-73, 2014.

[9] DE CARVAlHO FER, F. G. B.; LIMA, R. A. A Desvinculação das Receitas da União (DRU) como Instrumento de Flexibilização do Orçamento Público no Brasil: Necessidade ou Distorção? Revista de Direito Tributário e Financeiro, v. 2, n. 2, p. 309-330, 2017.

[10] DE OLIVEIRA, I. G. S. Análise de correspondência aplicada a preposições sobre a eficiência e eficácia do processo de compras públicas a partir da percepção de diferentes atores envolvidos (Public procurement in brazilian universities: analysis of correspondence on applied to prepositions efficiency and effective ness from different players involved perception). Revista Ciências Administrativas ou Journal of Administrative Sciences, v. 21, n. 1, 2015.

[11] FARIA, A. C.; COSTA, M. F. G. Gestão de custos logísticos: custeio baseado em atividades (ABC), balanced scorecard (BSC), valor econômico agregado (EVA). São Paulo: Atlas, 2008. 
[12] FILGUEIRAS, L. Economia, política e o bloco no poder no Brasil. Revista Bahia Análise \& Dados, v. 27, n. 2, p. 147-173, 2018.

[13] FLEURY, P. F.; WANKE, P; FIGUEIREDO, K. F. Logística empresarial. São Paulo: Atlas, 2007.

[14] GALLMANN, F.; BELVEDERE, V. Linking service level, inventory management and warehousing practices: a case-based managerial analysis. Springer Science + Business Media, v.4, p.28-38, 2011.

[15] GIAMBIAGI, F. ALÉM, A. C. de Finanças públicas: teoria e prática no Brasil. $4^{\mathrm{a}}$ ed. atualizada. Rio de Janeiro, 2011.

[16] GOMES, E. G. M. Gestão por resultados e eficiência na administração pública: uma análise à luz da experiência do governo de Minas Gerais. Tese de Doutorado do Curso de Doutorado em Administração Pública e Governo da FGV/EAESP. 2009.

[17] GONÇALVES, R. dos R. Modelos de programação linear inteira mista para resolver problemas de otimização de sistemas de distribuição de energia elétrica radiais. Tese de Doutorado. Faculdade de Engenharia do Câmpus de Ilha Solteira UNESP, 2013.

[18] HAYES, B. E. Medindo a Satisfação do Cliente: Desenvolvimento e uso de questionários e métodos de análise estatística. São Paulo: Quality Press, 2008.

[19] KOVACS, E. P. Atributos dos Serviços Logísticos ao Cliente no Fornecimento de Embalagens para a Indústria de Bebidas. ANPAD, 2005.

[20] LUCHI, J. S. P; CARNEIRO, T. C. J. Análise de eficiência e eficácia do pregão presencial. In: Encontro Nacional de Engenharia de Produção - ENEGEP, XXVI, Fortaleza. Anais. Fortaleza: ENEGEP, 2006.

[21] MIGUEL, P. A. C. et al. Metodologia de pesquisa em engenharia de produção e gestão de operações. 2.ed. Rio de Janeiro: Elsevier, 2012.

[22] SANTOS, R. C. L. F. Orçamento público. Florianópolis: Departamento de Ciências da Administração / UFSC; Brasília: CAPES: UAB, 2011.

[23] SOARES, M.; SCARPIN, J. E. Controle interno na administração pública: avaliando sua eficiência na gestão municipal. Revista de Ciências Jurídicas, v. 14, n. $\quad 1,2015$.

[24] TRIVELATO, P. V. et al. Avaliação da eficiência na alocação dos recursos econômicos financeiros no âmbito hospitalar. RAHIS, v. 12, n. 4, 2015. 